\title{
Recurrent Ovarian Cancer
}

The majority of patients with ovarian cancer will respond to the current first-line standard chemotherapy, which consists of a platinum compound (usually carboplatin) and a taxane (usually paclitaxel). With the combination of state-of-the-art debulking surgery and state-of-the-art chemotherapy the median survival of patients with advanced disease is $\sim 44$ months [1]. Despite this progress in primary treatment of ovarian cancer in the past decade most patients will develop recurrent disease. Until today, in these cases there is no curative treatment. The objectives of second-line treatments are control of symptoms, minimal toxicity, a convenient mode of application, improvement of quality of life, and - last but not least - improvement of progression-free and overall survival.

It has become clear over the last decade that the likelihood of response to chemotherapy at the time of recurrence is directly proportional to the time between discontinuation of first-line chemotherapy and the confirmation of recurrent disease. The treatment-free and platinum-free intervals have been shown to strongly predict the chance of response to a second-line chemotherapy. Based on the data of Markman and Hoskins [2] patients with recurrent disease are divided into two subgroups: those with platinum-resistant disease and those with platinum-sensitive disease. Platinum-resistant disease is characterized by no response to prior platinum chemotherapy (best response no change or progressive disease) or progression within the first 6 months after the end of primary therapy. The median progression-free survival in this group of patients is $\sim 22$ weeks and the median survival $\sim 40$ weeks. There are various agents with moderate activity for those patients. Currently there are no data supporting a combination therapy for this group of patients. Platinum-sensitive disease is characterized by a response to prior platinum chemotherapy (at least partial response) and a progression-free survival of at least 6 months. The median progression-free survival is $\sim 40$ weeks and the median survival $\sim 60$ weeks. Recently it could be shown in a large phase III trial of the ICON and AGO collaborators that retreatment with paclitaxel plus platinum improves both progression-free (median 13 vs. 10 months) and overall survival (median 29 vs. 24 months) compared to conventional platinum-based chemotherapy [3]. Translated into clinical practice this means that there is a new standard in the treatment of patients with relapsed ovarian cancer relapsing at least after 6 months of being treatment-free: the combination of paclitaxel and platinum.

The phase I trial of Sehouli et al. [4] published in the current issue of ONKOLOGIE investigated the combination of topotecan and gemcitabine in patients with recurrent ovarian cancer. In 23 patients they administered 80 cycles of the combination of topotecan and gemcitabine at different dose levels. The dose-limiting toxicity in this well performed study was thrombocytopenia and neutropenia. This was only of minor clinical relevance in terms of infection rates and/or supportive care. It appears that treatment with the combination of topotecan and gemcitabine is feasible in patients with recurrent ovarian cancer. However, the main question is: can we integrate this regimen in current concepts of treatment of patients with recurrent ovarian cancer?

For patients with recurrence later than 6 months after completion of first-line therapy standard of care is the combination of paclitaxel plus platinum-based chemotherapy (usually carboplatin). Would it be ethically justifiable to withhold the standard of care from this group of patients? Certainly not. There are two possibilities to test for improved efficacy of treatment in this group of patients: the first trial design would be standard of care (platinum plus paclitaxel) vs. standard of care plus one or two new agents as triplet or sequential doublets. However, in first-line treatment of advanced ovarian cancer until now no triplet has been shown to be superior to platinum plus paclitaxel [5-7]. It therefore seems unlikely that this concept will work in recurrent ovarian cancer. And for a design of

\begin{tabular}{|c|c|}
\hline KARGER & (C) 2004 S. Karger GmbH, Freiburg \\
\hline $\begin{array}{l}\text { Fax +49 } 7614520714 \\
\text { E-mail Information@Karger.de } \\
\text { www.karger.com }\end{array}$ & $\begin{array}{l}\text { Accessible online at: } \\
\text { www.karger.com/onk }\end{array}$ \\
\hline
\end{tabular}

Prof. Dr. med. Jacobus Pfisterer 
sequential doublets we do not even have results of a phase III trial. The second trial design would be changing one of the two agents of standard of care and adding another active, not cross-resistant agent, for example carboplatin and paclitaxel as standard of care versus carboplatin/gemcitabine as experimental arm. In any case platinum-based chemotherapy must be given to those patients as part of their second-line therapy. A phase III trial testing this combination is ongoing, enrollment is completed and we are waiting for results. From a scientific point of view it is nearly impossible to incorporate the combination of topotecan and gemcitabine in treatment strategies for this group of patients based on the results of internationally accepted concepts in the second-line treatment of patients with platinum-sensitive recurrent ovarian cancer. In the group of patients with primary progression or with recurrence within the first 6 months after completion of firstline therapy with platinum and paclitaxel there are various agents with moderate activity including topotecan and gemcitabine with response rates between 15 and $20 \%$ given as monotherapy. Nevertheless, progression-free and overall survival is short for those patients. Until today no combination therapy has been shown to be superior to monotherapy in this group of patients. Is efficacy as improvement of progressionfree and overall survival the primary aim in this poor-prognosis patient group? Control of symptoms, minimal toxicities, a convenient mode of application and quality of life are of almost equal importance. It is unclear whether the concept of combination therapy in this group of patients will improve efficacy (progression-free and overall survival) or not. Combination therapy with topotecan and gemcitabine is likely to be more toxic than monotherapy, has a more complicated mode of application compared to monotherapy and we can speculate about the influence on quality of life in this poor-prognosis patient group. However, there is a possibility to test the concept of combination therapy with topotecan and gemcitabine in this group of patients. The results of further phase III trials yet to be conducted will answer whether the combination of topotecan and gemcitabine is superior to monotherapy and whether this effect would outweigh possible disad- vantages in toxicity, mode of application (hospitalization) and quality of life.

Summarizing, the study by Sehouli et al. [4] gives further evidence that many effective agents are available in recurrent ovarian cancer and indicates that not only one single-agent treatment might be possible, even though it might be difficult to transfer the results of phase I/II trials to current concepts of treatment of recurrent ovarian cancer. This also indicates that every patient with ovarian cancer should be treated within a clinical trial.

Jacobus Pfisterer, Kiel

\section{References}

1 du Bois A, Lück HJ, Meier W, Adams HP, Möbus V, Costa S, Bauknecht T, Richter B, Warm M, Schröder W, Olbricht S, Nitz U, Jackisch C, Emons G, Wagner U, Kuhn W, Pfisterer J: A randomized clinical trial of cisplatin/paclitaxel versus carboplatin/paclitaxel as first-line treatment of ovarian cancer. J Natl Cancer Inst 2003;95:1320-1329.

2 Markman M, Hoskins W: Responses to salvage chemotherapy in ovarian cancer: A critical need for precise definitions of the treated population. J Clin Oncol 1992;10:513-514.

3 The ICON and AGO Collaborators: Paclitaxel plus platinum based chemotherapy versus conventional platinum based chemotherapy in women with relapsed ovarian cancer: the ICON4 / AGO-Ovar-2.2 trial. Lancet 2003;361:2099-2106.

4 Sehouli J, Stengel D, Oskay G, Blohmer J, Kaubitzsch S, Lichtenegger W on behalf of the Ovarian Cancer Study Group of the Nord-Ostdeutsche Gesellschaft für Gynäkologische Onkologie (NOGGO): A dose-finding study of topotecan in combination with gemcitabine in the treatment of patients with recurrent ovarian cancer after failure of first-line chemotherapy with paclitaxel and platinum. Onkologie 2004:27:58-64.

5 du Bois A, Weber B, Pfisterer J, Goupil A, Wagner U, Barats J, Olbricht S, Mousseau M, Nitz U, Meden H: Epirubicin/paclitaxel/carboplatin (TEC) vs. paclitaxel/carboplatin (TC) in first-line treatment of ovarian cancer FIGO stages IIb-IV. Interim results of an AGO-GINECO Intergroup phase III trial. Proc Am Soc Clin Oncol 2001;20:805.

6 Pignata S, Deplacido S, Scambia G, Divagno G, Naglieri E, Vernaglia Lombardi A, Biamonte R, Marinaccio M, Guida T, Manzione L: Topotecan vs. nihil after response to carboplatin and paclitaxel in advanced ovarian cancer. Early results of the MITO-1 (Multicenter Italian Trials in Ovarian Cancer) study. Proc Am Soc Clin Oncol 2003:22:1791.

7 Pfisterer J, Lortholary A, Kimmig R, Weber B, du Bois A, Bourgeois H, Wagner U, Coudert B, Meier W, Costa S: Paclitaxel/carboplatin (TC) vs. paclitaxel/carboplatin followed by topotecan (TC-TOP) in first-line treatment of ovarian cancer FIGO Stages IIb-IV. Interim results of a Gynecologic Cancer Intergroup phase III trial of the AGO Ovarian Cancer Study Group and GINECO. Proc Am Soc Clin Oncol 2003;22:1793. 